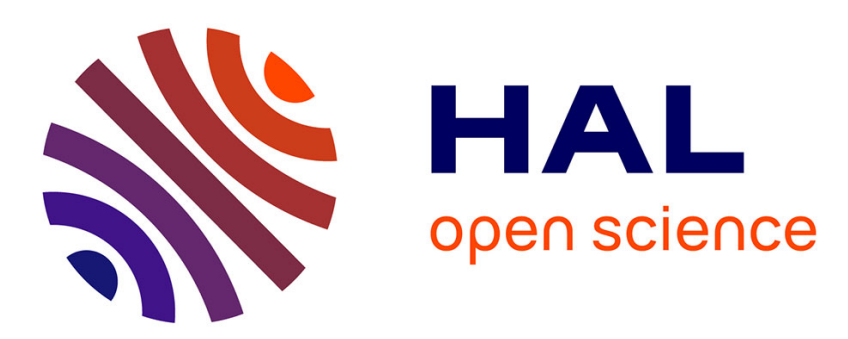

\title{
Molecular selectivity due to adsorption properties in nanotubes
}

\author{
Madjid Arab, F. Picaud, M. Devel, C. Ramseyer, C. Girardet
}

\section{To cite this version:}

Madjid Arab, F. Picaud, M. Devel, C. Ramseyer, C. Girardet. Molecular selectivity due to adsorption properties in nanotubes. Physical Review B: Condensed Matter and Materials Physics (1998-2015), 2004, 69 (16), pp.165401. 10.1103/PhysRevB.69.165401 . hal-01872987

\section{HAL Id: hal-01872987 \\ https://hal.science/hal-01872987}

Submitted on 26 May 2021

HAL is a multi-disciplinary open access archive for the deposit and dissemination of scientific research documents, whether they are published or not. The documents may come from teaching and research institutions in France or abroad, or from public or private research centers.
L'archive ouverte pluridisciplinaire $\mathbf{H A L}$, est destinée au dépôt et à la diffusion de documents scientifiques de niveau recherche, publiés ou non, émanant des établissements d'enseignement et de recherche français ou étrangers, des laboratoires publics ou privés. 


\title{
Molecular selectivity due to adsorption properties in nanotubes
}

\author{
M. Arab, F. Picaud, M. Devel, C. Ramseyer, and C. Girardet \\ Laboratoire de Physique Moléculaire, UMR 6624, Faculté des Sciences, La Bouloie, Université de Franche-Comté, \\ F25030 Besançon Cedex, France
}

(Received 31 October 2003; published 1 April 2004)

\begin{abstract}
The adsorption of small molecules $\left(\mathrm{N}_{2}, \mathrm{O}_{2}, \mathrm{CO}, \mathrm{CO}_{2}, \mathrm{H}_{2} \mathrm{O}\right.$, and $\left.\mathrm{HF}\right)$ in model ropes of carbon nanotubes has been studied to determine the main parameters (stable adsorption sites, potential barriers, ....) which define the ability of carbon nanotubes to select small molecules through their different behavior in the diffusion mechanism. When the polarization of the nanotubes is taken into account in the semiempirical potentials, it has a significant influence on the adsorption of polar species. Examination of the potential maps along the ropes shows that the nature and the stability of the adsorption sites are strongly dependent on the molecular species and on the diameter of the tubes. For a small rope formed with $(10,10)$ single-wall nanotubes, different trapping sites are favored by the molecules considered. Furthermore the corresponding trapping well depths are sufficiently selective to discriminate the species. Improving the size homogeneity of the ropes and judiciously calibrating their diameter would provide an efficient mean of selecting molecular species.
\end{abstract}

DOI: 10.1103/PhysRevB.69.165401

PACS number(s): 68.43.-h, 07.07.Df, 81.07.De

\section{INTRODUCTION}

Due to their importance in nanotechnology, carbon nanotubes (CNT's) have been intensively studied in recent years. ${ }^{1,2}$ Their excellent mechanical, electronic, and thermal properties offer wide potential applications ${ }^{3}$ in nanoelectronics, sensor probes, field emission displays or electron guns, for isolated tubes, or still in supercapacitors, electromechanical actuators, electromagnetic shielding or optical limiting material when included in composites. Single-wall nanotubes (SWNT) have recently attracted much attention regarding their use as miniature chemical or electrochemical sensor. ${ }^{4-11}$ Experimental reports have shown that upon exposure to gas molecules, the semiconducting SWNT's exhibit noticeable changes in their dielectric constant ${ }^{12,13}$ and very large changes in their electrical conductance. ${ }^{14-17}$ This high sensitivity to adsorption of molecules such as $\mathrm{O}_{2}, \mathrm{NO}_{2}$, and $\mathrm{NH}_{3}$ at room temperature has led to propose these SWNT's as sub ppm $(\approx 10 \mathrm{ppb}$ for some molecular species $)$ detectors ${ }^{18}$ considering that the sensor's scientific community is actually looking towards high performance new materials in environmental, industrial, and medical applications.

As all microporous materials, SWNT's are very useful for molecular sieving properties and they have been shown ${ }^{19,20}$ to display exceptional transport rates, with molecular fluxes that are orders of magnitude greater than crystalline zeolites for specific species such as $\mathrm{CH}_{4}$ and $\mathrm{H}_{2}$. In a more general way, information on the sensitivity of SWNT's as selective solvents of molecular species can be obtained from the determination of the adsorption energies on particular sites and of the energy corrugation along the nanotubes. A very small amount of experimental data is presently available mainly reporting measurements of isosteric heat of adsorption ${ }^{21,22}$ for a limited set of simple molecules adsorbed in/on heterogeneous SWNT bundles. First-principles methods ${ }^{11}$ using density functional at the local-density approximation (LDA) level have been applied to determine the adsorption energy of $\mathrm{NO}_{2}, \mathrm{O}_{2}, \mathrm{~N}_{2}, \mathrm{NH}_{3}, \mathrm{CO}_{2}, \mathrm{CH}_{4}$, and $\mathrm{H}_{2} \mathrm{O}$ on homogeneous bundles of SWNT having both zigzag or armchair structures. Full geometrical minimizations have been performed on various adsorption sites including nanotube surface, nanotube inner channels, grooves, and interstitial sites in ropes. The influence of the homogeneous vs heterogeneous distribution of nanotube diameters in the bundle has also been considered for $\mathrm{CH}_{4}$, $\mathrm{Ar}$, and Xe using Monte Carlo simulations with Lennard-Jones potential. ${ }^{23}$ It has been shown that the results of simulation are in excellent agreement with isosteric heats data for these species, when interstitial channels of defective NT bundles are taken into account in the calculations.

In the present paper, we consider the ability for SWNT bundles to separate some small molecules from a standard atmosphere, namely, water molecules and toxic gases such as $\mathrm{CO}$ (carbon monoxide), $\mathrm{CO}_{2}$ (carbon dioxide) or HF (fluoridric acid). For that purpose, we discuss results of adsorption/desorption energies and diffusion barriers. More specifically, we determine the relevant quantities, which will be used in a subsequent paper to analyze the dynamics and kinetics of the molecules moving through the bundle by kinetic Monte Carlo (KMC) simulations. Here the goal is first to design and test an accurate semiempirical potential by comparison with available density functional theory calculations. To build this potential, we add to the usual dispersionrepulsion contributions, the induction terms, which account for the nanotube polarization by the molecule electric moments. These latter terms will be shown to have a significant influence on the parameters governing the diffusion of the molecule, by favoring different adsorption sites for molecular species with different electrical properties. Then we analyze the influence of the nanotube size of the bundle confinement and of the molecular coverage (occurrence of molecular dimers) in the bundle on the relevant parameters.

The paper is organized as follows. In Sec. II, we describe the properties of the bundle used in the calculations, the interaction potential and the minimization procedure to reach the stable adsorption sites. Sec. III is devoted to the presentation of the results on the $0 \mathrm{~K}$ adsorption energy for typical 


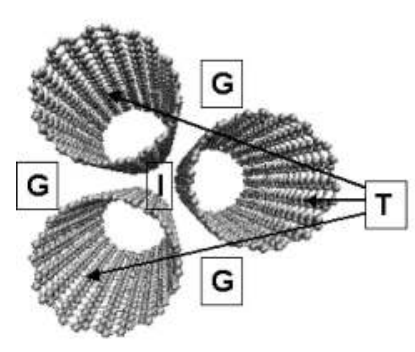

a)

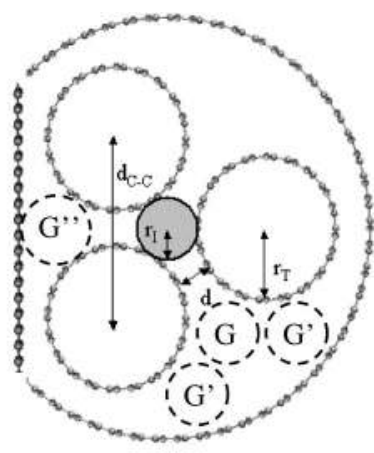

b)

FIG. 1. (a) Top view of a perfect bundle of nanotubes. The three most favorable adsorption sites are shown as $G$ for groove site, $T$ for an in-tube site, and $I$ for an interstitial site. The external site $E$ is also shown. The distance between each tube is equal to $3.4 \AA^{.24-26}$ (b) Top view of a confined bundle of nanotubes, the confinement is mimicked by the presence of a larger tube $(29,29)$, which is not deformed (right part of the figure) or, on the contrary, deformed (left part of the figure). In that confined configuration, new groove sites $G^{\prime}$ and $G^{\prime \prime}$ occur.

molecules $\mathrm{N}_{2}, \mathrm{O}_{2}, \mathrm{CO}, \mathrm{CO}_{2}, \mathrm{H}_{2} \mathrm{O}$, and $\mathrm{HF}$ and these data are compared to those issued from experiments and/or other theoretical papers. We discuss in Sec. IV the selectivity of various model bundles through the results of the adsorption energy and corrugation for isolated molecules and dimers.

\section{THEORETICAL BACKGROUNDS}

\section{A. Description of the system}

The adsorption of small molecules in or outside nanotubes is the key of our study. In order to optimize the sieving capacities of the sensor formed by SWNT we chose to simulate a bundle of tubes by putting a regular arrangement of tubes on a hexagonal array. More precisely, we consider opened carbon nanotubes to increase the possibilities for the adsorbed molecules to be kept inside tube or to pass through the nanotube array. In a first configuration, the model bundle is formed by three identical $(10,10)$ tubes [cf Fig. 1(a)] since this is the smallest cluster required to represent all the possible sites in the hexagonal lattice. We use this system to study the adsorption energy in the different sites accessible to the molecules, namely, the in-tube site $T$, groove site $G$, and interstitial site $I$ which are described more precisely in Fig. 1(a). We will also consider the adsorption site $E$ on the external surface of a tube to allow a comparison with available data. The distance between each $(10,10)$ tube in the array is set to $3.4 \AA$, i.e., the interlayer distance between graphite planes. ${ }^{24-26}$ In a second study, we vary the radius and interspace of the tubes, nonetheless keeping the hexagonal structure and we evaluate the influence of the size of the sites $T$, $I$, and $G$ on the adsorption properties of the molecules. This is a simple way to simulate an heterogeneous bundle without explicitly introducing a size distribution for the tubes. Finally, in the third situation, the three $(10,10)$ tubes are enclosed in a larger $(29,29)$ tube (simulation of a mesopore system), deformed or not, to mimic the influence of confine-

ment on the adsorption sites and the diffusion of the molecules, which could be due to the presence of a matrix around the bundle.

The molecules considered hereafter are those frequently met in the atmosphere $\left(\mathrm{N}_{2}, \mathrm{O}_{2}\right.$, and $\left.\mathrm{H}_{2} \mathrm{O}\right)$. An interest of this paper is to study the behavior of those latter molecules in comparison with air pollutants such as $\mathrm{CO}$ and $\mathrm{CO}_{2}$, and the much more harmful and toxic HF molecule in order to assess whether or not such bundles can serve to energetically separate $\mathrm{CO}, \mathrm{CO}_{2}$, and $\mathrm{HF}$ molecules from the ambient atmosphere using adsorption and diffusion criteria.

\section{B. Polarization of nanotubes}

Polarization effects of SWNT are expected to influence significantly the adsorption of molecules. Under an external electric field, SWNT's can be bent (or twisted) simply because of mutual interaction of each induced dipole created on carbon atoms. ${ }^{27}$ To account for these polarization effects, we describe in a first approximation each carbon atom by an isotropic atomic polarizability $\alpha_{C}=1.2 \AA^{3}$. This value is the current polarizability used to describe the electric properties of fullerenes. Recently it has been shown ${ }^{28,29}$ that the mutual polarization interaction between carbon atoms in nanotubes is not constant but depends on the length of the tubes. Using a method based on the resolution of the Lippman-Schwinger equation and anisotropic atomic polarizabilities to describe the propagation of the induction in a mesoscopic tube, ${ }^{30}$ the effective carbon atom polarizability in a tube has been found to increase with the length of the tube up to some coherency length $^{31}$ that could be of the order of few micrometers for real metallic nanotubes. We assume that those length effects will not disrupt the general potential energy curves obtained by moving the molecule throughout the bundle. Indeed, we have verified in a test case that, compared to the isotropic polarizability model, these mutual effects of exaltation between carbon polarizabilities are responsible for a small displacement in energy (at most $30 \mathrm{meV}$ ) without appreciably changing the shape of the potential maps. This justifies the neglect of atomic anisotropic polarizabilities and non-selfconsistent resolution which leads to a very important gain of CPU time in simulations.

\section{Interaction between adsorbed molecules and nanotubes}

\section{Electrical field created by a molecule on a carbon atom}

The total electric field created by the molecule on the tubes is written as a sum of contributions $E^{(n)}$ due to charge $(n=0)$, dipole $(n=1)$, and quadrupole $(n=2)$ distributed on different sites of the molecule. The electrostatic description of each molecule is summarized in Table I. These sites are generally located on the nuclei and at the middle of the bonds for the diatomic molecules. ${ }^{32,33}$ Within this approach, the electric field $E$ created by the molecule $j$ on the $c_{t h}$ carbon atom in the tube at a mutual distance $r_{j c}$ is defined as: ${ }^{32}$

$$
\mathbf{E}\left(r_{j c}\right)=\sum_{i}\left(\mathbf{T}_{0}^{i} \mathbf{M}_{0}^{i}-\mathbf{T}_{1}^{i} \mathbf{M}_{1}^{i}+\frac{1}{3} \mathbf{T}_{2}^{i} \mathbf{M}_{2}^{i}\right)
$$


TABLE I. Electrostatic description of the molecules. The interaction centers that bear the various electric poles $M_{n}$ can be the atoms themselves $(\mathrm{C}, \mathrm{N}, \mathrm{F}, \mathrm{O}, \mathrm{H})$ or the molecular center of mass (c.m.) or still the middle $(M)$ of the bond depending on the description of the interaction potential. All data are given in atomic units.

\begin{tabular}{|c|c|c|c|c|c|}
\hline Molecule & Site & Position $(\AA)$ & $M_{0}(e)$ & $M_{1}(e \AA)$ & $M_{2}\left(e \AA^{2}\right)$ \\
\hline \multirow[t]{5}{*}{$\mathrm{CO}_{2}$} & $C$ & 0 & 1.17 & 0 & -0.143 \\
\hline & $O$ & 1.16 & -0.31 & 0.053 & 0.048 \\
\hline & $M$ & 0.58 & -0.28 & -0.042 & 0.185 \\
\hline & $O$ & -1.16 & -0.31 & 0.053 & 0.048 \\
\hline & $M$ & -0.58 & -0.28 & -0.042 & 0.185 \\
\hline Molecular electric pole & c.m. & 0 & 0 & 0 & -1.049 \\
\hline \multirow[t]{3}{*}{$\mathrm{O}_{2}$} & $O$ & -0.607 & 0 & 0 & -0.081 \\
\hline & $M$ & 0 & 0 & 0 & 0.162 \\
\hline & $O$ & 0.607 & 0 & 0 & -0.081 \\
\hline Molecular electric pole & c.m. & 0 & 0 & 0 & 0 \\
\hline \multirow[t]{3}{*}{$\mathrm{N}_{2}$} & $N$ & -0.547 & 0.60 & 0.428 & -0.025 \\
\hline & $M$ & 0 & -1.20 & 0 & 0.381 \\
\hline & $N$ & 0.547 & 0.60 & -0.428 & -0.025 \\
\hline Molecular electric pole & c.m. & 0 & 0 & 0 & -0.246 \\
\hline \multirow[t]{3}{*}{$\mathrm{CO}$} & $C$ & -0.644 & 0.63 & 0.459 & -0.160 \\
\hline & $M$ & -0.080 & -0.72 & 0.0529 & 0.207 \\
\hline & $O$ & 0.484 & 0.09 & -0.259 & 0.098 \\
\hline Molecular electric pole & c.m. & 0 & 0 & -0.053 & -0.420 \\
\hline \multirow[t]{3}{*}{$\mathrm{HF}$} & $F$ & -0.046 & -0.07 & 0.164 & 0.168 \\
\hline & $M$ & 0.412 & -0.54 & -0.079 & 0.011 \\
\hline & $H$ & 0.870 & 0.61 & 0.05 & 0.017 \\
\hline Molecular electric pole & c.m. & 0 & 0 & 0.400 & 0.491 \\
\hline $\mathrm{H}_{2} \mathrm{O}$ & c.m. & 0 & 0 & 0.385 & $\begin{array}{c}M_{2 x x}=0.548 \\
M_{2 y y}=-0.520 \\
M_{2 z z}=-0.027\end{array}$ \\
\hline
\end{tabular}

where $T_{n}\left(r_{i c}\right)$ defines the interaction tensor associated with the $n_{t h}$ multipole $M_{n}$ located at the $i_{t h}$ site of the molecule. The response of any carbon atom to this electric field gives rise to an induced dipole moment, which is written in a first approximation, as:

$$
\boldsymbol{\mu}_{i n d}=\sum_{i} \boldsymbol{\alpha}_{C} \times \mathbf{E}\left(r_{j c}\right)
$$

where $\alpha_{C}$ is the isotropic polarizability of carbon atom in the tube, taken to be $1.2 \AA^{3} .^{34,35}$ These induced dipoles on $\mathrm{C}$ atoms are assumed not to interact, in contrast, with more refined theories including the polarization in a self-consistent way, as already mentioned.

\section{Potential-energy calculations}

The interaction potential between a molecule and all the carbon atoms of the bundle is expressed as a sum of two contributions, namely:

$$
V^{m o l-B}=\sum_{c}\left[V_{D R}\left(r_{j c}\right)+V_{I}\left(r_{j c}\right)\right]
$$

When several molecules are adsorbed in/on the tubes, we have to add to Eq. (3), the molecule-molecule interaction:

$$
V^{m o l-m o l}=\sum_{j, j^{\prime}}\left[V_{D R}\left(r_{j j^{\prime}}\right)+V_{E}\left(r_{j j^{\prime}}\right)\right]
$$

where $V_{D R}$ represents the quantum interaction (dispersion and repulsion terms) either between the $j_{t h}$ molecule and the carbon atom separated by a distance $r_{j c}$ [in Eq. (3)], or between two molecules at a distance $r_{j j^{\prime}}$ [in Eq. (4)]. These interactions are expressed in terms of pairwise atom-atom Lennard-Jones (LJ) potentials:

$$
V_{D R}^{L J}=\sum_{i, i^{\prime}} 4 \epsilon_{i i^{\prime}}\left(\frac{\sigma_{i i^{\prime}}^{12}}{r_{i i^{\prime}}^{12}}-\frac{\sigma_{i i^{\prime}}^{6}}{r_{i i^{\prime}}^{6}}\right)
$$

where $\epsilon_{i i^{\prime}}, \sigma_{i i^{\prime}}$ are the usual energy and diameter LJ parameters and $r_{i i^{\prime}}$ is the distance between two atoms $i$ and $i^{\prime}$ belonging to two different molecules or an atom of the molecule $i$ and a carbon $i^{\prime} \equiv j_{C}$ of the bundle. These LJ parameters are given in Table II for the carbon-molecule pairs.

Using the Buckingham formalism, ${ }^{36}$ the induction potential $V_{I}\left(r_{j c}\right)$ in Eq. (3) on the carbon atoms and the electrostatic molecule-molecule potential $V_{E}\left(r_{i j}\right)$ in Eq. (4) have the same following form: 
TABLE II. Lennard-Jones parameters between a carbon atoms in a tube and the molecules.

\begin{tabular}{lccc}
\hline \hline & Atom-atom & $\epsilon(\mathrm{meV})$ & $\sigma(\AA)$ \\
\hline $\mathrm{C}-\mathrm{CO}_{2}$ & $\mathrm{C}-\mathrm{C}$ & 2.10 & 3.50 \\
& $\mathrm{C}-\mathrm{O}$ & 3.10 & 3.23 \\
$\mathrm{C}-\mathrm{O}_{2}$ & $\mathrm{C}-\mathrm{O}$ & 3.10 & 3.23 \\
$\mathrm{C}-\mathrm{N}_{2}$ & $\mathrm{C}-\mathrm{N}$ & 2.63 & 3.44 \\
$\mathrm{C}-\mathrm{CO}$ & $\mathrm{C}-\mathrm{C}$ & 2.10 & 3.50 \\
& $\mathrm{C}-\mathrm{O}$ & 3.10 & 3.23 \\
$\mathrm{C}-\mathrm{HF}$ & $\mathrm{C}-\mathrm{H}$ & 2.10 & 2.79 \\
& $\mathrm{C}-\mathrm{F}$ & 2.65 & 3.61 \\
$\mathrm{C}-\mathrm{H}_{2} \mathrm{O}$ & $\mathrm{C}-\mathrm{H}$ & 2.10 & 3.01 \\
& $\mathrm{C}-\mathrm{O}$ & 3.10 & 3.23 \\
\hline \hline
\end{tabular}

$$
V(r)=\sum_{n, m} \sum_{j, i \epsilon j} \sum_{j^{\prime}, i^{\prime} \epsilon j^{\prime}} \mathbf{M}_{j_{i}}^{n} \mathbf{T}^{n+m}\left(\mathbf{r}_{j_{i} j^{\prime}{ }^{\prime}}\right) \mathbf{M}_{j^{\prime}{ }^{\prime}}{ }^{\prime} m
$$

where the sums are all over the electrostatic sites of the molecules (or the carbon atoms) in the tube. The dipole moment tensor $M^{1}$ attached to the carbon atom is induced by the molecular moments, while for the adsorbed molecules, $M^{0}, M^{1}$, and $M^{2}$ are permanent multipoles. The induction contribution between two molecules remains small in general and has been neglected with respect to the electrostatic contribution in $V^{m o l-m o l}$ [Eq. (4)].

\section{Energy optimization}

The stable configuration for the adsorbed molecule is determined from a conjugate-gradient procedure speeded up by using the analytical expressions of the derivatives. For each system, the total potential $V$ is minimized with respect to the position and the orientation of the adsorbed molecules. The carbon tubes are assumed to be rigid and undeformable. We draw the potential energy map $V_{m}(z)$ by minimizing $V$ with respect to the $(x, y)$ position and $(\theta, \phi, \chi)$ orientation of the molecule for a fixed height $z$ of the molecular center of mass. The $z$ axis is assumed to be along the symmetry axis of the tubes and we studied the evolution of the energy map with $z$ for the adsorbed molecules. A large sampling of initial positions and orientations of the molecules allows us to reach accurate equilibrium diffusion valleys in the bundle, without artificial trappings into local minima. As it will be shown, the minimum search depends on the sensitivity of the potential to small position and orientation changes, and on the accuracy of the parameters used in these calculations.

\section{RESULTS}

\section{A. Ideal bundles formed by $(\mathbf{1 0 , 1 0 )}$ SWNT}

\section{Non-Polar Molecules : $\mathrm{CO}_{2}, \mathrm{O}_{2}, \mathrm{~N}_{2}, \mathrm{CO}$}

The adsorption energy of small nondipolar (or very weakly dipolar for $\mathrm{CO}$ ) molecules in an ideal bundle formed by $(10,10)$ SWNT's is first determined in order to test the accuracy of the interaction potential $V^{m o l-B}$. Such molecules which are present in the atmosphere as dominant elements or as pollutants are mainly quadrupolar and they will poorly interact through induction terms with the carbon atoms of the tubes. Thus a comparison (Sec. IV) with available experimental data for $\mathrm{CO}_{2}, \mathrm{O}_{2}, \mathrm{~N}_{2}, \mathrm{CO}$ appears to be a test of the Lennard-Jones potential used to describe the dispersionrepulsion interactions between carbon atoms and those molecules.

In preliminary calculations, we have verified that the adsorption energy at $0 \mathrm{~K}$ does not depend on the length of the tubes by varying the number of carbon atoms from 800 , 1040,1440 up to 1800 . All the results will therefore be discussed using $(10,10)$ tubes containing 1040 carbon atoms. We present in Table III the results of our calculations with the corresponding available data issued from calculations or from experiments. While the stable orientation of the four molecules is the same, namely, with their axis pointing along the direction of the tube axis, the type of the most stable adsorption site is different. Indeed, the $G, I$, and $T$ sites have close energies for $\mathrm{CO}_{2}$, with however a slightly smaller value for the interstitial site, whereas the external site $E$ is clearly much less stable by about $100 \mathrm{meV}$. The interstitial site is the most strongly attractive for $\mathrm{O}_{2}$ while the two adsorption sites ( $T$ and $G$ ) are less stable and nearly equivalent and the external site $E$ close in energy to the $\mathrm{CO}_{2}$ one, i.e., much less stable than the other sites. For $\mathrm{N}_{2}$ and $\mathrm{CO}$, we find two equivalently stable sites $G$ and $T$, and two much less stable sites $I$ and $E$. Further discussion of the differences between our results and the other data quoted is postponed to Sec. IV.

\section{Dipolar Molecules: $\mathrm{HF}, \mathrm{H}_{2} \mathrm{O}$}

Since $\mathrm{HF}$ and $\mathrm{H}_{2} \mathrm{O}$ molecules are strongly dipolar and quadrupolar, the polarization effects due to the interaction between these multipole moments and the dipoles induced on carbon atoms by the electric field of the permanent multipoles of the molecules (induction contribution) are considerably larger than for the nondipolar species. In contrast to these latter molecules, the molecular axis of $\mathrm{HF}$ and the $\mathrm{C}_{2}$ axis of $\mathrm{H}_{2} \mathrm{O}$ bearing the dipolar moment tend to point perpendicular to the tube axis towards the carbon atoms. For $\mathrm{HF}$, the stable adsorption sites are the $T$ and $G$ sites with close energy values. The other two sites $I$ and $E$ are much less stable. In the most stable sites, the induction energy accounts for about $45 \%$ of the total energy, while this contribution was totally negligible for $\mathrm{O}_{2}$ or $\mathrm{N}_{2}$. For $\mathrm{H}_{2} \mathrm{O}$, the most stable site is the $I$ site while the $G$ and $T$ sites, and $a$ fortiori the $E$ site, have much higher adsorption energies. In the $I$ site, the induction contribution represents $38 \%$ of the total energy.

\section{B. Non ideal SWNT bundles}

While bundles formed by a single species of SWNT appear as a theoretical idealization, experimental adsorption studies of molecules on nanotubes are performed on NT's with randomly distributed sizes, lengths, and orientations. Studying the influence of random distribution of NT sizes requires to vary the radius of the tubes in the bundle and their mutual distance. Therefore, we have calculated the ad- 
TABLE III. Calculated adsorption energy $(V)$ at $0 \mathrm{~K}$ in the various sites (see the text) and comparison with available experimental $\left(V_{E X P}\right)$ and theoretical $\left(V_{M M}\right.$ for molecular mechanics calculations or $V_{L D A}$ for local-density approximation) data.

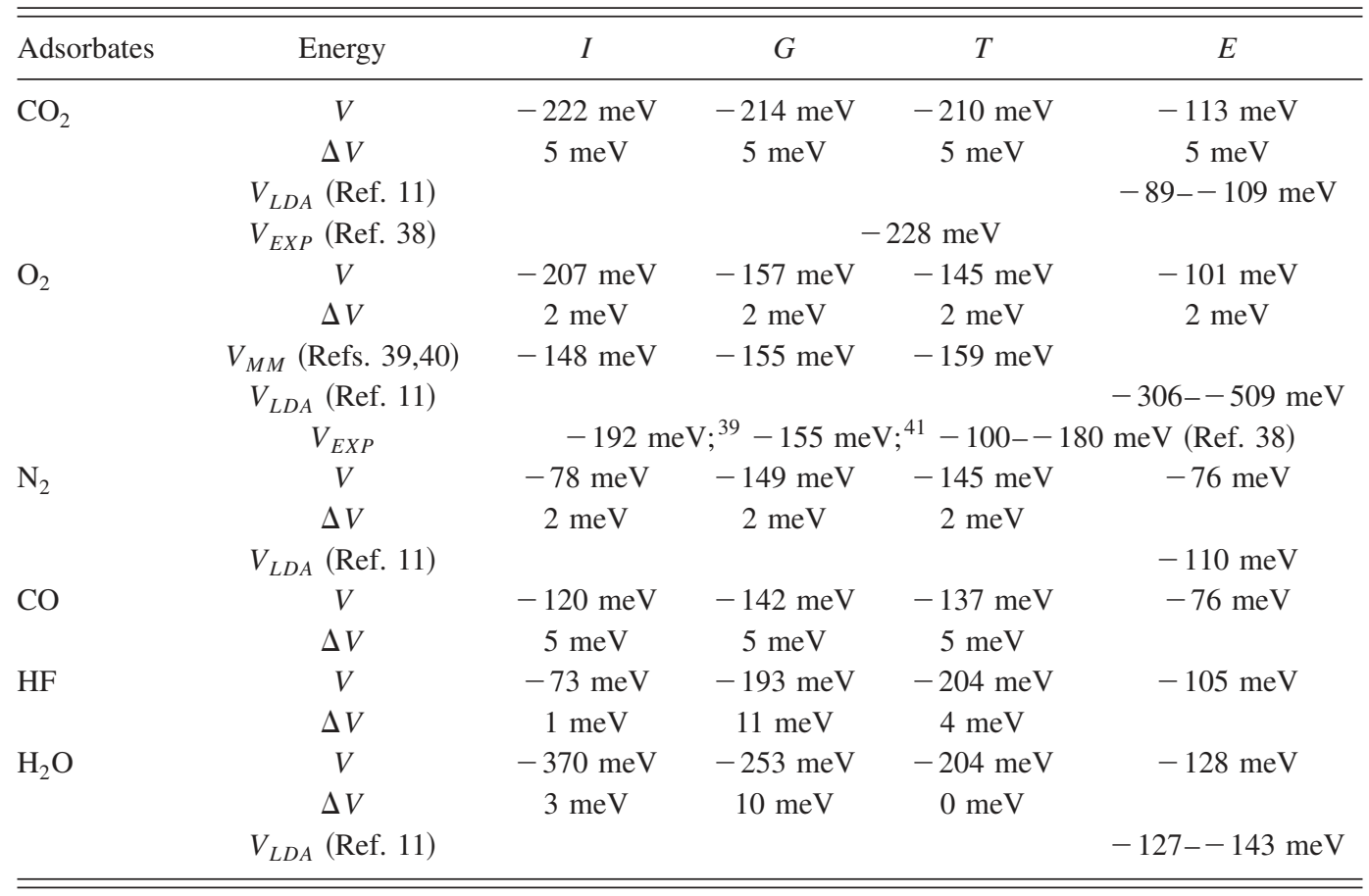

sorption energy at $0 \mathrm{~K}$ of the three molecules $\mathrm{N}_{2}, \mathrm{HF}$, and $\mathrm{H}_{2} \mathrm{O}$ as a function of a single relevant parameter $r_{I}$ representing the radius of the interstitial channel formed by three NT's when their radius $r_{T}$ and their mutual distance $d$ are changed. $r_{I}$ is a linear function of $r_{T}$ and $d$, as

$$
r_{I}=\left(\frac{2-\sqrt{3}}{\sqrt{3}}\right) r_{T}+\frac{d}{\sqrt{3}}
$$

which can thus be directly related to the size of the tubes, and in a less direct way, to the size of the groove (Fig. 1). Figure 2 shows the behavior of the adsorption energy of the three molecules trapped in the stable adsorption sites $T, I$, and $G$ with the values of $r_{I}$. Since the distance $d$ is generally defined with a rather small standard deviation $d=3.4$ $\pm 0.3 \AA$, the variations of $r_{I}$ will be mainly correlated with the variations of the tube radius. Note that for an ideal bundle formed by $(10,10)$ tubes with $r_{T}=6.8 \AA$ (Sec. III A), the value of $r_{I}$ is equal to $3.0 \AA$, when $d=3.4 \AA$. To evaluate the potential-energy evolution of each site as a function of $r_{I}$, two ways have been chosen. First, we have studied the potential energy in site $T$ when $r_{T}$ is varied and then transposed it into a function of $r_{I}$ using Eq. (7). Second, the potential energies for sites $G$ and $I$ have been calculated as a function of $d$ and then plotted vs $r_{I}$ from Eq. (7).

We see in Fig. 2 that the curves of adsorption energy vs $r_{I}$ corresponding to the adsorption sites $T$ and $I$ display a single minimum, which is much sharper for the internal site $T$ than for the interstitial site $I$. In site $T$, the energy is minimum for relatively small radii of the tubes $\left(r_{T}=2.8,3.4\right.$, and $4.1 \AA$ for $\mathrm{N}_{2}, \mathrm{H}_{2} \mathrm{O}$, and $\mathrm{HF}$, respectively). In site $I$, the energy minima are found for $r_{I}=3.2,3.1$, and $3.3 \AA$ for the three molecules in the same sequence. The different shape of the potential maps (cf. Fig. 3 showing the potential map for $\mathrm{H}_{2} \mathrm{O}$ ), with quasicylindric or triangular symmetries for the $T$ or $G$ and $I$ sites explains why the $r_{T}$ values are more dispersed than the $r_{I}$ values. The energy curves for the groove site have a more regular behavior with a very flat minimum. This can be understood in the present model by the fact that the molecules can move much more freely in the groove sites (no constraint outside the bundle) to keep their energy constant and minimum. A more complete discussion of this situation will be given in Sec. III C.

Four domains for the energy behavior can be distinguished in Fig. 2. When $r_{I}$ is small, i.e., $r_{I}<2.8-2.9 \AA, T$ is the most stable site for the three molecules. This corresponds to values of $r_{T}$ ranging between 5.4 and $6.1 \AA$. For $r_{I}$ values between 2.8 and $3.1 \AA$, the $G$ site becomes the most stable in a very narrow domain (for $\mathrm{H}_{2} \mathrm{O}$ this domain is nearly reduced to values around $2.8 \AA$ ). Increasing the $r_{I}$ values $\left(3.1 \leqslant r_{I} \leqslant 4.5 \AA\right)$ changes the stable site which becomes the $I$ site. At still larger values of $r_{I}\left(r_{I} \geqslant 4.50 \AA\right)$, the $G$ and $I$ sites have similar energies for the two polar molecules, while site $I$ remains the most stable for $N_{2}$ whatever $r_{I}$ values. It is particularly striking that the narrow domain $2.8-3.1 \AA$ corresponds to values of $r_{T}$ including the $(10,10)$ tube radius which appears to be among the most probable species in the experimental distribution of tubes in a bundle. ${ }^{37} \mathrm{~A}$ similar behavior is found for the other molecules $\mathrm{O}_{2}, \mathrm{CO}$, and $\mathrm{CO}_{2}$ (not given here).

Finally, let us note that the attractive adsorption energies found for molecules with large sigma parameter (about $3.5 \AA$ ) inside interstitial channel (of radius $3 \AA$ ) is a priori surprising but can be explained differently in the nonpolar 


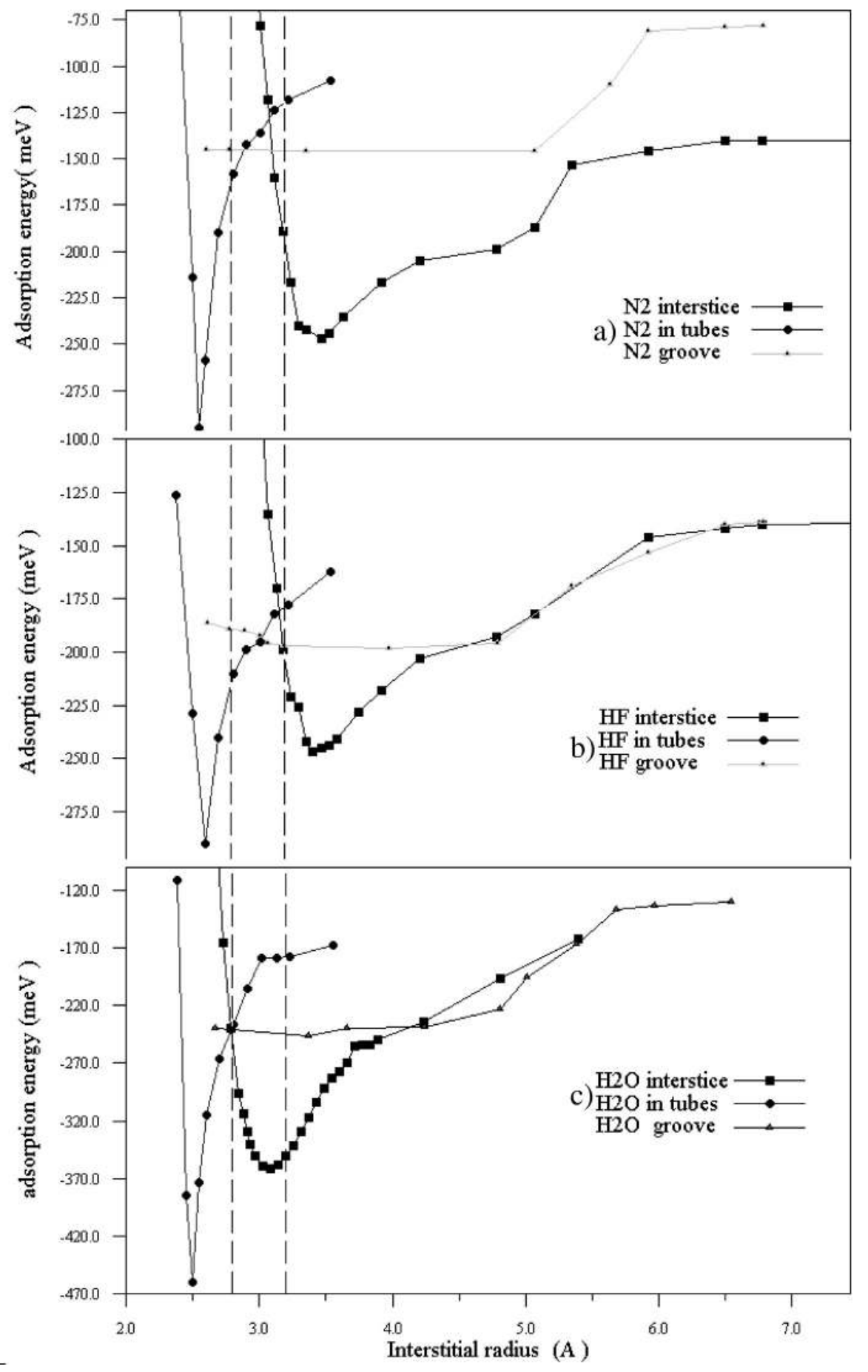

FIG. 2. Adsorption energy at 0 $\mathrm{K}$ of three molecules $\left(\mathrm{N}_{2}, \mathrm{HF}\right.$, and $\left.\mathrm{H}_{2} \mathrm{O}\right)$ vs the intertistial radius $r_{I}(\AA)$ defined in the three channels $I, T$, and $G$. The vertical broken lines represent the experimental domain for which $r_{I}$ can exist.

and polar cases. For nonpolar molecules, this attractive energy is only due to $\mathrm{LJ}$ potential. In the case of the $\mathrm{CO}_{2}$ molecule, we have checked that the repulsive contribution given by the $12 \mathrm{C}$ atoms nearest to the molecule is more than compensated by the large amount of small contributions coming from the other $\mathrm{C}$ atoms of the 3 nanotubes. For HF molecules, the phenomenon is completely different since the effects of polarization contribute for $-120 \mathrm{meV}$ to the total energy whereas the $\mathrm{LJ}$ potential is equal to $+40 \mathrm{meV}$. This stresses the importance of including polarization effects when studying the adsorption of small molecules with permanent dipolar moment.

\section{Encapsulated ideal SWNT bundle}

Since one of the goal of this paper is to determine the ability for SWNT to selectively sieve small molecules through their diffusion parallel to the tube axis along the $T, I$, and $G$ sites of the bundle, we discuss the encapsulated model as described in Sec. II A. When the $(29,29)$ tube, which encapsulates the bundle is not distorted [right part of Fig. 1(b)], the energy of the $G$ site is exactly the same as for the free bundle. In addition two symmetric energy wells named $G^{\prime}$ occur between the $(10,10)$ and $(29,29)$ tubes. In Fig. 3, the potential map drawn for $\mathrm{H}_{2} \mathrm{O}$ displays the characteristic shapes experienced by the molecule exploring the bundle, 


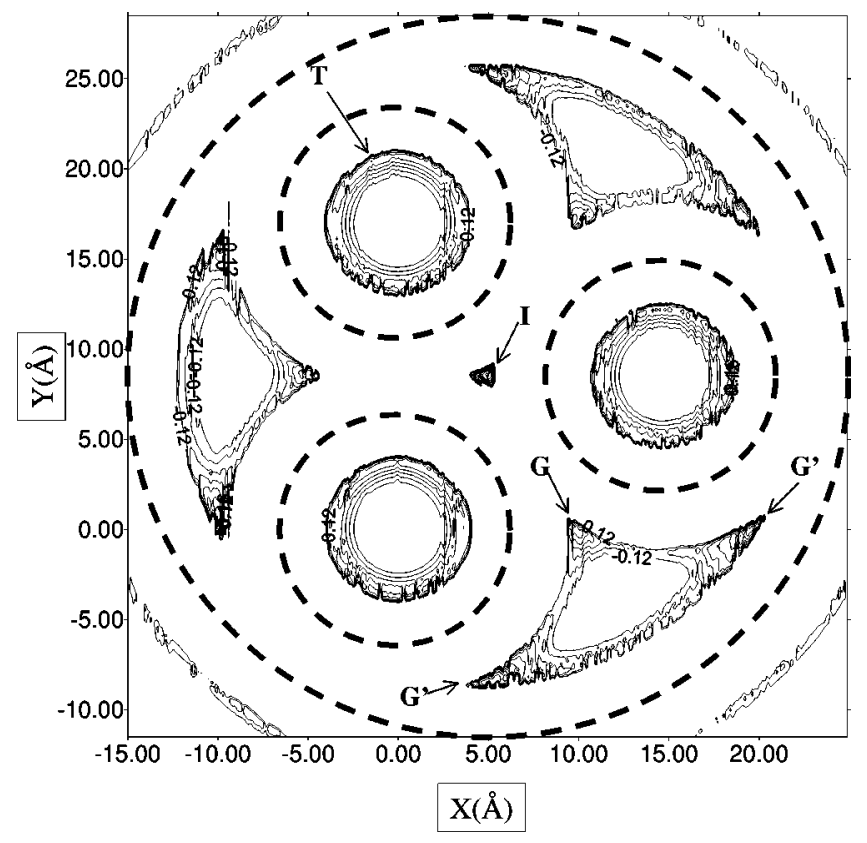

FIG. 3. Potential-energy map for $\mathrm{H}_{2} \mathrm{O}$ molecule inside an encapsulated ideal SWNT bundle. Only isoenergy curves corresponding to values less than $-120 \mathrm{meV}$ are drawn. The circular curves correspond to internal sites $T$, the spherical triangles to groove sites $G$ or $G^{\prime}$ and the small triangle at the center of the figure to interstitial site $I$. The small broken circles are drawn in superimposition to indicate the walls of each $(10,10)$ tube and the big broken circle indicate the $(29,29)$ tube position.

with the triangular shape of the site $I$, the circular shape inside each tube and still a triangular shape for the confined groove sites. Note that the minimum isopotential curve does not correspond to the tube or triangle centers but rather to the external part of each geometric figure (triangle or circle). These wells $G^{\prime}$ are slightly more attractive (by about 15 $\mathrm{meV}$ ) than the original $G$ sites for the three molecules considered here due to the influence of the confinement (cf. Fig. $2)$. Introducing a relatively abrupt distortion of the $(29,29)$ tube as described in Sec. II A, leads to the disappearance of the $G^{\prime}$ sites and the reoccurrence of a single site named $G^{\prime \prime}$ [left part of Fig. 1(b)] with a well significantly deeper by about $50 \mathrm{meV}$, for the three molecules, due to the influence of the $(29,29)$ tube. It can be noted that encapsulation of the bundle modifies the shape and size of the $G$ sites but it does not change the stability of the various sites found for the perfect $(10,10)$ tube bundle: The $G$ site appears to be the most stable for $\mathrm{N}_{2}$ and $\mathrm{HF}$ while $\mathrm{H}_{2} \mathrm{O}$ prefers to be adsorbed in interstitial site $I$.

This encapsulated bundle formed by three $(10,10)$ tubes containing each 1040 atoms inside a $(29,29)$ tube of the same length has been chosen as a model to determine the parameters which will be used in forthcoming KMC calculations. These calculations will describe the diffusion ability of the six molecular species considered in terms of energy barriers required for each molecule to enter the bundle through the $T$, $I$, and $G\left(G^{\prime}\right.$ and $\left.G^{\prime \prime}\right)$ sites and to diffuse inside the channels formed by these sites along the tube axis. Generally, our calculations show that the corrugations in the various chan- nels remain very small, as indicated by the values reported in Table III. It does not exceed $11 \mathrm{meV}$. However, the energy barrier preventing the entry to some channels of the bundle varies significantly with the molecular species, or from one site to another site [Fig. 4(a)]. While $\mathrm{CO}_{2}, \mathrm{O}_{2}$, and $\mathrm{H}_{2} \mathrm{O}$ can enter freely (no barrier) inside the channels defined by the three sites species, a barrier of $77 \mathrm{meV}$ for $\mathrm{HF}, 65 \mathrm{meV}$ for $\mathrm{N}_{2}$, and $40 \mathrm{meV}$ for $\mathrm{CO}$ prevents these molecules to enter site $I$ in the bundle [Fig. 4(b)]. Note that increasing the value of $r_{I}$, and thus of $r_{T}$, leads to the occurrence of a small barrier (about $20 \mathrm{meV}$ ) for $\mathrm{N}_{2}$ and $\mathrm{HF}$ entrance via the $I$ site, while no barrier is found via the $G$ site.

\section{Influence of molecular interactions in an ideal bundle}

To complete our study on the parameters, which will characterize the efficiency of SWNT bundles acting as molecular sieves, let us analyze whether the presence of a second molecule of the same species, through formation of a dimer adsorbed in the same channel, can influence the results obtained for a single molecule. While there is no significant influence on the corrugation in a given channel, the interaction between two molecules in a nearest-neighbor position could be strongly modified by the competition between the orientational dependence of the molecule-molecule and molecule-bundle potentials. We give in Table IV the interaction energy between two molecules in the gas phase (last column of Table IV) compared with the interaction energy between the same molecules trapped in their stable sites $(G$, $I$, or $T$ depending on the molecule) when the total interaction energy $V^{m o l-C}+V^{m o l-m o l}$ is minimized with respect to the orientations and the positions of the two molecules. For HF and $\mathrm{H}_{2} \mathrm{O}$, there is in general no significant misorientation of the molecular axes for most of the sites and molecular species. Furthermore the interaction energy in the dimer remains close to the value found for the gas phases. By contrast, the I site destabilizes, entirely, the orientation of the molecules in the confined dimers $(\mathrm{CO})_{2},\left(\mathrm{CO}_{2}\right)_{2},\left(\mathrm{O}_{2}\right)_{2}$, and $\left(\mathrm{N}_{2}\right)_{2}$ with a concomitant repulsive dimer energy. Indeed, the molecules in the dimers become mutually colinear and parallel to the tube axis, instead of being mutually perpendicular for $\mathrm{O}_{2}$ and $\mathrm{N}_{2}$ and the centers of mass are mutually translated along the tube axis for $\mathrm{CO}$ and $\mathrm{CO}_{2}$ (Table IV). On the basis of these results, we see that the orientational stability of the dimers in vacuum is generally kept when they are confined in their most favorable channels. Therefore the potential barrier hindering the dimer escape towards the gas phase is approximatively twice the barrier for the monomer while the barrier preventing the molecule escape from a dimer breakness of the dimer bond and from the bundle is enhanced by half the mutual interaction in the dimer. Such an enhancement is particularly significant for the polar species (increase by about $50 \%$ of the barrier height) but smoother for the nondipolar molecules (increase by 10 to $15 \%$ only). Forthcoming calculations are in progress to study the formation and behavior of nanowires inside the bundle since it is expected that several molecules should be simultaneously adsorbed inside the channels. 

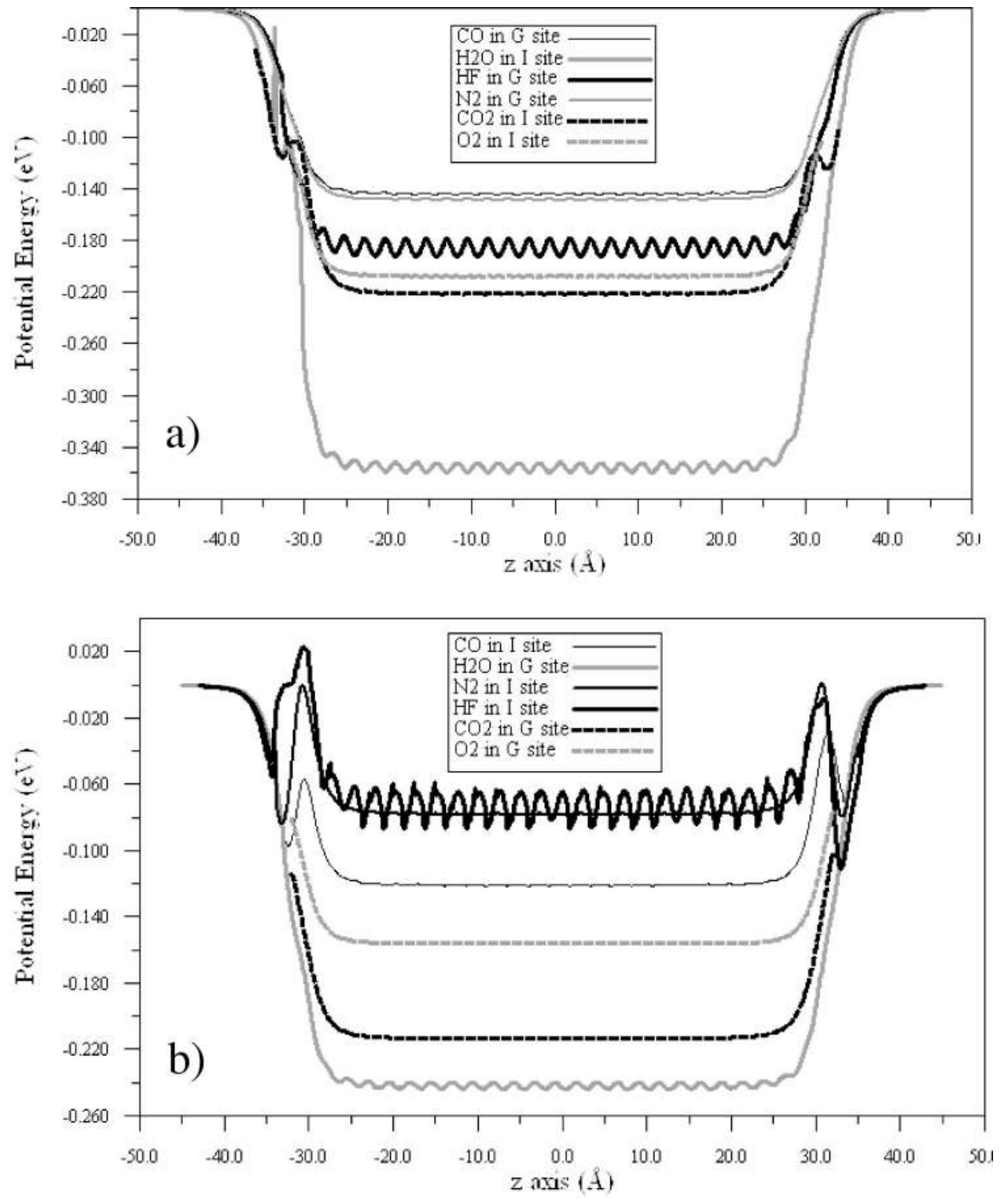

FIG. 4. (a) Potential-energy diffusion valleys along a bundle formed by three $(10,10)$ tubes (65 $\AA$ long) for the most favorable molecular adsorption sites (site $I$ for $\mathrm{CO}_{2}, \mathrm{O}_{2}$, and $\mathrm{H}_{2} \mathrm{O}$ and site $G$ for $\mathrm{CO}, \mathrm{N}_{2}$, and $\mathrm{HF}$ ). (b) Potential-energy diffusion valleys along a bundle formed by three $(10,10)$ tubes ( $65 \AA$ long) for adsorption sites which are kinetically accessible, though less stable.

\section{DISCUSSION}

\section{A. Comparison with available data on the adsorption energy}

We first focus on the accuracy of the molecule-SWNT interaction potential by comparing our results with available theoretical and experimental data (Table III). Using adsorption isotherm measurements, Bienfait et al. ${ }^{38}$ have recently determined the adsorption energy of $\mathrm{CO}_{2}$ in SWNT bundles formed by a distribution of nanotube diameters centered around the $(10,10)$ size. The measured energy, in good agreement with our calculations, was assigned to $\mathrm{CO}_{2}$ adsorption in $I$ and $G$ sites without any further discrimination. The $T$ site was excluded, based on the hypothesis that most of the tubes were closed in the bundle. No other information about this molecule was available, excepted for the $\mathrm{CO}_{2}$ adsorption at the surface of single nanotubes. Zhao et al. ${ }^{11}$ found, using LDA calculations, an adsorption energy varying from -89 to $-107 \mathrm{meV}$, depending on the position (on top of a carbon atom, in a bridged position or in a hollow site) of the $\mathrm{CO}_{2}$ molecule. This latter value is fully consistent with our results for site $E$.
For $\mathrm{O}_{2}$, the experimental data in the literature are issued either from thermal desorption ${ }^{39,40}$ or from adsorption isotherm measurements. ${ }^{38,41}$ The value obtained from thermal desorption $(-191 \mathrm{meV})$ is in very good agreement with the values calculated in the $I$ sites, while the data of adsorption isotherms lead to slightly larger values, from $-155 \mathrm{meV}$ for Wei et al. ${ }^{41}$ to $-180 \mathrm{meV}$ for Bienfait et al. ${ }^{38}$ in good agreement with our results on $T$ and $G$ sites. Besides, these latter authors assigned this energy to $\mathrm{O}_{2}$ adsorption in $G$ site, and found another energy $(-110 \mathrm{meV})$, which could correspond to adsorption at the outer rounded surface of a tube, very close to the value calculated in site $E$. The available theoretical data appear to be much more dispersed. Molecular mechanical calculations ${ }^{40}$ lead to energy values very close in $T, G$, and $I$ sites (respectively $-159,-155$, and $-148 \mathrm{meV})$. Note that we find a more strongly attractive site $I$ for this molecule, in contrast with this latter result but in a realistic range of energy. Using $a b$ initio calculations performed within LDA method, Zhao et al. ${ }^{11}$ found an energy varying from -306 to $-509 \mathrm{meV}$ on the external sur- 
TABLE IV. Dimer energies and configurations determined for the various channels of the bundle and in vacuum. $\Theta$ characterizes the angle defining the mutual orientation of the molecular axes in the dimer. A geometric view of the stable configurations for the dimers in the channels is shown for each case.

\begin{tabular}{|c|c|c|c|c|c|c|c|c|}
\hline \multirow[t]{2}{*}{ Molecules } & \multicolumn{2}{|c|}{ Site G } & \multicolumn{2}{|c|}{ Site I } & \multicolumn{2}{|c|}{ Site T } & \multicolumn{2}{|c|}{ Yacuum } \\
\hline & $\mathbf{E}_{\mathrm{G}(\mathbf{m e V})}$ & $\theta_{G^{(0)}}$ & $\mathbf{E}_{\mathbf{1}}(\mathbf{m e V})$ & $\left.\theta_{1^{(}}{ }^{\circ}\right)$ & $\mathbf{E}_{\mathrm{T}}(\mathrm{meV})$ & $\theta_{\left.\mathrm{T}^{(}\right)}$ & $\mathbf{E}_{\mathrm{v}}(\mathrm{meV})$ & $\theta_{v}\left({ }^{\circ}\right)$ \\
\hline $\mathrm{CO}_{2}$ & -55 & 10 & rep. & $0 ?$ & -61 & 3 & -64 & \\
\hline $\mathrm{O}_{2}$ & -12 & 8 & -13 & 5 & -30 & $82 ?$ & -33 & 87 \\
\hline $\mathrm{N}_{2}$ & -31 & $102 ?$ & rep. & 5 & -31 & $106 !$ & -33 & 99 ! \\
\hline $\mathrm{CO}$ & -32 & 10 & rep. & $1 \xi$ & -32 & 15 & -34 & 10 \\
\hline HF & -237 & 108 & -231 & $\begin{array}{r}114 \\
\vdots \\
\vdots\end{array}$ & -236 & 110 & -240 & 120 \\
\hline $\mathrm{H}_{2} \mathrm{O}$ & -272 & 45 & -282 & 45 & -289 & 45 & -290 & $\begin{array}{l}45 \\
\rightarrow\end{array}$ \\
\hline
\end{tabular}

face of a tube. Based on the same type of calculations, Sorescu et al., ${ }^{42}$ Peng et al., ${ }^{43}$ and Jhi et al. ${ }^{44}$ determined binding energies for $\mathrm{O}_{2}$ which range between -38 and $-250 \mathrm{meV}$, with a significant charge transfer from the tube to $\mathrm{O}_{2}$ molecule in some cases. This latter phenomenon is probably at the origin of such a dispersion in the energy values.

The results for $\mathrm{N}_{2}$ in sites $G$ and $T$ are also in very good agreement with the average binding energy determined from adsorption isotherms by Wei et al. ${ }^{41}$ while the adsorption on the external surface of a tube appears to be $30 \%$ in error with respect to the value calculated with LDA (Ref. 11) for $\mathrm{N}_{2}$ on graphene. Unfortunately, no data are available for the adsorption energy of $\mathrm{CO}$ and $\mathrm{HF}$, and values are only known from LDA method ${ }^{11}$ for $\mathrm{H}_{2} \mathrm{O}$ adsorbed on the external surface. These latter values, ranging between -145 and $-127 \mathrm{meV}$ according to $\mathrm{H}_{2} \mathrm{O}$ is adsorbed in top, bridge, or hollow sites are fully consistent with our calculations in site $E$.

To summarize, this comparison, though unfortunately partial due to the lack of previous information for some molecules, shows an overall satisfactory agreement of our results with experimental data which can justify the use of semiempirical potentials in forthcoming simulations.

\section{B. Parameters for the molecule diffusion in bundle}

To discuss the role of SWNT bundles as a filter for small molecules, we consider the ideal bundle made of $(10,10)$ tubes. The common feature for all the molecules considered here is the very small corrugation along the various channels in the bundle, at most $11 \mathrm{meV}$ for $\mathrm{HF}$, when compared to the thermal energy at $300 \mathrm{~K}\left(k_{B} T=25 \mathrm{meV}\right)$. We thus expect that the molecular diffusion will be governed by the friction regime (space or energy limited diffusion models) inside the channels, and by a possible potential barrier at the entrance and the large potential barrier at the exit of the bundle. We have shown that the most stable site for $\mathrm{O}_{2}$ and $\mathrm{H}_{2} \mathrm{O}$ is the site $I$ in the ideal bundle while it is the site $G$ for $\mathrm{N}_{2}, \mathrm{CO}$, and $\mathrm{HF}$ (for $\mathrm{CO}_{2}$ the three sites $I, G$, and $T$ are very close in energy). Since all these molecules do not experience an energy barrier at the entrance of the bundle, we conclude that $\mathrm{O}_{2}$ and $\mathrm{H}_{2} \mathrm{O}$ (and in a less extent $\mathrm{CO}_{2}$ ) will not occupy the same channels than the other molecules. In other words, within the thermodynamic regime approximation an energetic selectivity of the molecules occur via the stability of the sites. It can be noted that experimental data have shown that $\mathrm{O}_{2}$ and $\mathrm{N}_{2}$ could prefer the $G$ site while adsorption isotherm measurements for $\mathrm{CO}_{2}$ have led to the conclusion that the $G$ and $I$ sites would not be distinguishable. Unfortunately, we have no experimental information regarding the preferential adsorption for $\mathrm{H}_{2} \mathrm{O}$ and $\mathrm{HF}$.

From a kinetic point of view, regarding the potential barriers, which prevent the molecules to leave the ideal bundle channels corresponding to their most stable sites, they can be identified at $0 \mathrm{~K}$ with the adsorption energies (Table III). Their heights vary from about $150 \mathrm{meV}$ for $\mathrm{CO}$ and $\mathrm{N}_{2}$, to $200 \mathrm{meV}$ for $\mathrm{HF}$ and $\mathrm{CO}_{2}$ and reach $370 \mathrm{meV}$ for $\mathrm{H}_{2} \mathrm{O}$. Molecular-dynamics (MD) simulations followed by kinetic Monte Carlo calculations will define precisely the energy selectivity of the ideal bundle using the barrier height data. However, the corrugation felt by any molecules between adsorption sites along the tube axis direction is in fact very low compared to the exit barriers and to the thermal energy at ambient temperature (about $25 \mathrm{meV}$ ). This points towards an easy diffusion of all the molecules considered here, along the tubes. On the contrary, a real competition between each molecules indeed appears when considering the exit probability: If one uses a very simple version of the diffusion probability described by an Arrhenius law and the same prefactor for every molecule, the time selectivity follows the barrier height ordering [Fig. 4(a)], i.e., desorption of $\mathrm{CO}$ and $\mathrm{N}_{2}$ first and then $\mathrm{HF}, \mathrm{CO}_{2}, \mathrm{O}_{2}$, and finally $\mathrm{H}_{2} \mathrm{O}$. 
Note that the $T$ sites are also easily accessible to the molecules without any barrier at the tube entrance, provided the $(10,10)$ tubes are opened ${ }^{45}$ and keep their bulk symmetry at their extremities. Indeed, although they are not the most stable sites, free entrance and only very small corrugation are found in $T$ channels for all the molecular species studied here. According to several experimental discussions, the presence of oxygen, which tends to oxidize the tube extremities, has been shown to play an important role on the opening and closing of the tube extremities. ${ }^{45}$ As a result, the tubes seem to remain opened over too short durations to allow the passage of the molecules. However tubes opened by ball milling seem to stay open over long times and for temperatures well above room temperature in absence of strong oxidizing species. ${ }^{46}$ Therefore, the possibility for the molecules to diffuse through $T$ site channels cannot be a priori excluded.

A similar remark regarding the site accessibility can be done for $\mathrm{CO}, \mathrm{N}_{2}$, and $\mathrm{HF}$ in the $I$ site channels, and for $\mathrm{O}_{2}$ and $\mathrm{H}_{2} \mathrm{O}$ in the $G$ site channels. Indeed, although less stable, these channels could be kinetically possible, for $\mathrm{O}_{2}$ and $\mathrm{H}_{2} \mathrm{O}$ without any barrier at the entrance, and for $\mathrm{CO}, \mathrm{N}_{2}$ and $\mathrm{HF}$ with energy barrier heights around 50-70 meV [Fig. 4(b)]. The occurrence of these barriers can be understood by the reorientation of the three molecules axes $\mathrm{CO}, \mathrm{N}_{2}$, and $\mathrm{HF}$ at the extremities of the bundle due to electrostatic effects, while $\mathrm{H}_{2} \mathrm{O}$, by changing its configuration at the entrance of the bundle, does not experience any constraint to be adsorbed in site $I$.

When the size of the tubes in the bundle is varied, we have seen that the stability of the sites can change from site $T$ to site $I$ when the tube radius increases. At intermediate radii the $G$ site can become the most stable adsorption position. Figure 2 gives us a complete overview of preferential adsorption for the considered molecular species whatever the tube size in the bundle. It is specially striking that most of the experimental synthesized bundles display a mean distance between nearest-neighbor tubes equal to $17.0 \pm 1.0 \AA$. It corresponds to a value for $r_{I}=3.0 \pm 0.2 \AA$, which is about exactly the range of radii for which the $G$ site becomes the most stable in our calculations.

When the bundle is encapsulated in a larger $(29,29)$ tube, the overall results are not changed, except for an increase of the well depth. For $\mathrm{N}_{2}$ and HF, the $G^{\prime}$ and $G^{\prime \prime}$ sites substitute to $\mathrm{G}$ and they appear slightly deeper. For $\mathrm{H}_{2} \mathrm{O}$, the $G$ site energy is also enhanced but this site remains less stable than site $I$. When the encapsulated bundle is drastically deformed, no inversion in the energy diagram can be found in our calculations. The $G$ site is still the most attractive well for $\mathrm{N}_{2}$ and $\mathrm{HF}$, whereas $\mathrm{H}_{2} \mathrm{O}$ prefers to adsorb inside site $I$.

To end this discussion, a homogeneous bundle formed by $(10,10)$ SWNT's appears to provide some energy selectivity in terms of barrier heights for the molecule escape from NT's. $\mathrm{H}_{2} \mathrm{O}$ and $\mathrm{O}_{2}$ molecules have a singular behavior regarding their most stable site $I$ with respect to the other species which prefer the $G$ site. The $\mathrm{H}_{2} \mathrm{O}$ molecule is also singular by the barrier height which is much larger than for the other species. In contrast, the HF molecule does not display such properties and it cannot be discriminated so easily, at least at this step of the calculations. Decreasing (increasing) the size of the NT's to open the possibility of new stable sites and thus to mimic the bundle heterogeneity shows that site $I$ (T) can become more stable for most of the considered molecules at $0 \mathrm{~K}$. However these results should be supported by $\mathrm{MD}$ and KMC simulations and they should also account for the number of available sites in a given bundle. This number for the $I, G$, and $T$ sites inside a hexagonal bundle can be written in terms of the numbers $n$ of tubes forming one side of the hexagon, as

$$
\begin{gathered}
n_{I}=6(n-2)^{2}, \\
n_{T}=3 n(n-2)+1, \\
n_{G}=6(n-2) .
\end{gathered}
$$

For typical experimental bundles ${ }^{38}$ the number of tubes $n_{T}$ generally varies from 30 to 50 leading to $n \simeq 5$ or 6 . The corresponding values for $n_{I}$ and $n_{G}$ are $n_{I}=54$ to 96 and $n_{G}=18$ to 24 . It is thus clear that the $I$ sites are predominant, and in terms of probability, they would behave as favorable channels for $\mathrm{H}_{2} \mathrm{O}$ and $\mathrm{O}_{2}$ with large trapping barriers. For the other molecular species, the competition between kinetics and thermodynamics requires additional calculations.

\section{CONCLUSION}

We have studied the adsorption of small molecules present in ambient atmosphere as dominant elements $\left(\mathrm{N}_{2}\right.$, $\left.\mathrm{O}_{2}\right)$, pollutants $\left(\mathrm{CO}, \mathrm{CO}_{2}, \mathrm{H}_{2} \mathrm{O}\right)$ or even traces $(\mathrm{HF})$, in order to provide information on the ability for these bundles to behave as selective molecular sensors, through their trapping and sieving properties. The aim was to characterize the physical quantities (adsorption wells, corrugation, barrier heights, and aggregation traps), which will be used in molecular dynamics or kinetic Monte Carlo simulations. All these quantities are issued from the knowledge of the molecule-NT interaction potential which should offer the best compromise between accuracy and simplicity (for reasonable CPU times). The first results show that (i) including polarization effects in the interaction potential is required for the polar molecules, (ii) the sieving properties of SWNT's depend strongly on the radius of the tubes and four domains of behavior regarding the most stable site can be found depending on the radius values, (iii) the corrugation inside the bundle is very small, and (iv) $\mathrm{H}_{2} \mathrm{O}, \mathrm{CO}_{2}$, and $\mathrm{O}_{2}$ prefer interstitial sites while the groove sites are more favorable energetically for $\mathrm{CO}, \mathrm{N}_{2}$, and HF. Selectivity in terms of barrier heights for escaping the groove sites can be efficient regarding the stronger trapping of HF compared to $\mathrm{CO}$ and $\mathrm{N}_{2}$. Molecular-dynamics and kinetic Monte Carlo calculations are required to go beyond this preliminary analysis.

\section{ACKNOWLEDGMENTS}

The authors express their thanks to F. Berger, M. Bienfait, and J.M. Vigoureux for fruitful discussions. One of us (M.A.) would like to acknowledge the hospitality of CRMC2 at Marseille for one week, which welcomed him to perform isotherm adsorption experiments. 
${ }^{1}$ R. Saito, M. S. Dresselhaus, and G. Dresselhaus, Physical properties of carbon nanotubes (Imperial College Press, London, 1998).

${ }^{2}$ M. S. Dresselhaus, G. Dresselhaus, and P. Avouris, Carbone Nanotubes: Synthesis, Structure, Properties, and Applications, Springer Topics in Applied Physics Vol. 80 (Springer, Berlin, 2001).

${ }^{3}$ R.H. Baughman, A.A. Zhakidov, and W.A. de Heer, Science 297, 787 (2002).

${ }^{4}$ J. Kong, N. Franklin, C. Zhou, M. Chapline, S. Peng, K. Cho, and H. Dai, Science 287, 622 (2000).

${ }^{5}$ A. Dillon, K. Jones, T. Bekkedahl, C. Klang, D. Bethune, and M. Heben, Nature (London) 386, 377 (1997).

${ }^{6}$ Q. Wang, S. Challa, D. Sholl, and J. Johnson, Phys. Rev. Lett. 82, 956 (1999).

${ }^{7}$ A. Ramirez-Pastor, T. Eggarter, V. Pereyra, and J. Riccardo, Phys. Rev. B 59, 11027 (1999).

${ }^{8}$ G. Stan, M. Bojan, M. Curtarolo, S. Gatica, and M. Cole, Phys. Rev. B 62, 2173 (2000).

${ }^{9}$ M. Cole, V. Crespi, G. Stan, C. Ebner, J. Hartman, S. Moroni, and M. Boninsegni, Phys. Rev. Lett. 84, 3883 (2000).

${ }^{10}$ S. Gatica, M. Bojan, G. Stan, and M. Cole, J. Chem. Phys. 114, 3765 (2001).

${ }^{11}$ J. Zhao, A. Buldum, J. Han, and J. Pinglu, Nanotechnology 13, 195 (2002).

${ }^{12}$ S. Chopra, A. Pham, J. Gaillard, A. Parker, and A. Rao, Appl. Phys. Lett. 80, 4632 (2002).

${ }^{13}$ S. Chopra, K. McGuire, N. Gothard, A. Rao, and A. Pham, Appl. Phys. Lett. 83, 2280 (2003).

${ }^{14}$ L. Valentini, L. Lozzi, C. Cantalini, I. Armentano, J. Kenny, and S. Santucci, Thin Solid Films 436, 95 (2003).

${ }^{15}$ C. Cantalini, L. Valentini, L. Lozzi, I. Armentano, J. Kenny, and S. Santucci, Sens. Actuators B 95, 195 (2003).

${ }^{16}$ S. Peng, J. O'Keefe, C. Wei, K. Cho, J. Kong, R. Chen, N. Franklin, and H. Dai, Conference Proceedings of the 3rd International Workshop on Structural Health Monitoring (Stanford, CA, 2001).

${ }^{17}$ P. Giannozzi, R. Car, and G. Scholes, J. Chem. Phys. 118, 1003 (2003).

${ }^{18}$ C. Adu, G. Sumanasekera, B. Pradhan, H. Romero, and P. Eklund, J. Chem. Phys. 337, 31 (2001).

${ }^{19}$ Y. Ye, C. Ahn, C. Witham, B. Fultz, J. Liu, A. Rinzler, D. Colbert, K. Smith, and R. Smalley, Appl. Phys. Lett. 74, 2307 (1999).

${ }^{20}$ C. Liu, Y. Fan, M. Liu, H. Cong, H. Cheng, and M. Dresselhaus, Science 286, 1127 (1999).
${ }^{21}$ A. Kleinhammes, S. Mao, X. Yang, X. Tang, H. Shimoda, J. Lu, O. Zhou, and Y. Wu, Phys. Rev. B 68, 075418 (2003).

${ }^{22}$ W. Teizer, R. Hallock, E. Dujardin, and T. Ebbesen, Phys. Rev. Lett. 82, 5305 (1999).

${ }^{23}$ W. Shi and J. Johnson, Phys. Rev. Lett. 91, 015504 (2003); M.R. Johnson, S. Rols, P. Waas, M. Muris, M. Bienfait, P. Zeppenfeld, and N. Dupont-Pavlosky, Chem. Phys. 293, 217 (2003).

${ }^{24}$ U. Venkateswaran, A. Rao, E. Richter, M. Menon, A. Rinzler, R. Smalley, and P. Eklund, Phys. Rev. B 59, 10928 (1999).

${ }^{25}$ S. Maruyama and T. Kimura, International Mechanical Engineering Congress and Exhibit (Orlando, U.S.A., 2000).

${ }^{26}$ T. Werder, J. Walther, and P. Koumoutsakos, Nanotech. 2, 490 (2002).

${ }^{27}$ P. Poncharal, Z.L. Wang, D. Ugarte, and W.A. de Heer, Science 283, 1513 (1999).

${ }^{28}$ H. Kim, M. Cole, F. Toigo, and D. Nicholson, Surf. Sci. 198, 555 (1988).

${ }^{29}$ L.X. Benedict, S.G. Louie, and M.L. Cohen, Phys. Rev. B 52, 8541 (1995).

${ }^{30}$ M. Devel, C. Girard, and C. Joachim, Phys. Rev. B 53, 13159 (1996).

${ }^{31}$ R. Langlet, M. Devel, and J. M. Vigoureux (unpublished).

${ }^{32}$ A. Stone, The theory of intermolecular forces (Clarandon, Oxford, 1996).

${ }^{33}$ A. Stone and M. Alderton, Mol. Phys. 56, 1047 (1985).

${ }^{34}$ L. Jensen, O. Schmidt, P. Astrand, and K. Mikkelsen, J. Phys. Chem. B 104, 10462 (2000).

${ }^{35}$ L. Jensen, P. Astrand, A. Ostred, J. Kongsted, and K. Mikkelsen, J. Chem. Phys. 116, 4001 (2002).

${ }^{36}$ A. Buckingham, Adv. Chem. Phys. 12, 107 (1967).

${ }^{37}$ D. Bethune, Physica B 323, 90 (2002).

${ }^{38}$ M. Bienfait, P. Zeppenfeld, N. Dupont-Pavlosky, M. Muris, M. Johnson, T. Wilson, M. de Pies, and O. Vilches (unpublished).

${ }^{39}$ H. Ulbricht, G. Moos, and T. Hertel, Surf. Sci. 532-535, 852 (2003).

${ }^{40}$ H. Ulbricht, G. Moos, and T. Hertel, Phys. Rev. B 66, 075404 (2002).

${ }^{41}$ B. Wei, M. Hsu, Y. Yang, S. Chien, and H. Lin, Mater. Chem. Phys. 81, 126 (2003).

${ }^{42}$ D. Sorescu, K. Jordan, and P. Avouris, J. Phys. Chem. B 105, 11227 (2001).

${ }^{43}$ S. Peng and K. Cho, Nanotechnology 11, 57 (2000).

${ }^{44}$ H. Jhi, S. Louie, and M. Cohen, Phys. Rev. Lett. 85, 1710 (2000).

${ }^{45}$ M. Babaa, I. Stepanek, K. Masenelli-Varlot, N. Dupont-Pavlosky, E. McRae, and P. Bernier, Surf. Sci. 531, 86 (2003).

${ }^{46} \mathrm{M}$. Babaa (private communication). 